\title{
Application of Improved Analysis of Convective Heat Loads on Plasma Facing Components to Wendelstein 7-X
}

\author{
Daniel Böckenhoff ${ }^{1}$, Marko Blatzheim ${ }^{1,2}$ and the W7-X Team ${ }^{1}$ \\ ${ }^{1}$ Max Planck Institute for Plasma Physics, Wendelsteinstraße 1, 17491 Greifswald, Germany \\ ${ }^{2}$ Institute for Mathematics, University of Rostock, Ulmenstraße 69, 18057 Rostock, Germany
}

daniel.boeckenhoff@ipp.mpg.de,marko.blatzheim@ipp.mpg.de

\begin{abstract}
Within this research we present a method to speed up the simulation of convective heat loads onto the plasma-facing components (PFCs) of the Wendelstein 7-X (W7-X) stellarator by a factor of approximately 20 with the same statistical precision as compared to the previous standard. The geometric models developed for this are also designed to unravel the complex 3D PFCs onto a 2D picture-like input format which gives access to the full set of image analysis tools like for example wavelet analysis or the applicability of convolutional neural network (CNN) architectures.

The significant speedup of heat load calculation allows to simulate a massive data set of heat loads for approximately $3 \times 10^{4}$ magnetic configurations in edge rotational transform-radial axis shift space. To first order, plasma dynamic effects like toroidal current development as well as beta effects mainly influence this space which motivates the simulation in this scope.

A criterion to evaluate the safety of a magnetic configuration with respect to the convective heat load onto the plasma facing components has been developed taking statistical fluctuations of the simulation into account. This criterion, applied to the introduced data set, provides a map relevant for discharge planning and machine safety.

The methods and concepts introduced herein could contribute to the safety evaluation of magnetic confinement devices in general and are not specific for W7-X.
\end{abstract}

\section{Introduction}

Wendelstein 7-X (W7-X) is a stellarator type nuclear fusion experiment [1, 2]. Three main goals were defined for the project. The first, demonstrating the feasibility of building a superconducting modular stellarator with the required precision, has been reached, as shown by the many successful experiments conducted already 3 5. A second objective is to demonstrate the accessibility of plasma parameters close to those of a future fusion power plant, which is ongoing work [6 $[9]$. The last is to prove the possibility of continuous operation [6]. To accomplish this, it is essential to protect the plasma facing components (PFCs) from heat loads exceeding the design specifications. We refer to this case as 'overload'.

Monte Carlo field line diffusion simulation is the standard tool to calculate the heat load onto the PFCs. It is a heavily used method for discharge planning and evaluation. In order to obtain PFC heat loads with statistical significance, it has so far been necessary to simulate with $\mathcal{O}\left(10^{6}\right)$ single field line diffusion traces, resulting in run times of roughly 10 hours. Due to evolving plasma density, temperature and currents, the PFC heat load is dynamic [5, $10-12]$ and multiple simulations have to be performed. This can result in days to weeks of simulation time.

With this work, we introduce new geometrical models of the most important W7-X PFCs, i. e. the divertor, baffle and heat shield. The new models have the same geometric fidelity as the original models but are partitioned in a way that introduces the following beneficial properties:

a) They reduce the necessary field line diffusion simulation time by a factor of 20 with the same or higher statistical significance. Due to the significant decrease in simulation time, high resolution multi-dimensional magnetic configuration scans become feasible.

b) The new partitions take into account physics and engineering constraints of the respective PFCs.

c) They can be mapped to a two dimensional data format well suited for heat load display and analysis. This mapping is not restricted to simulation results but also applicable to experimental heat loads calculated from Infra-Red (IR) temperature data [13]. Notably, the format allows the applica- 
tion of convolutional neural network (CNN) architectures to the problem of PFC heat load feature extraction since it is equivalent to a picture-like array, preserving neighborhood relations between the "pixels". To be very clear: In the scope of the present article no artificial neural networks (NNs) or other machine learning (ML) techniques are applied although they are envisioned. So far heat load visualizations have either been realized by a camera-matrix projection, where the complex curved geometry is distorted 14,15 , or by one-dimensional heat load quantification along a predefined trajectory 15.

Furthermore, a new method is introduced that allows a probabilistic assessment of the overload evaluation for a given magnetic field and a power convected along this field. This method considers each particle trace of a field line diffusion simulation as a Bernoulli process 16].

A high resolution two-dimensional magnetic configuration scan in rotational transform $t$ and radial shift $\Delta R$ with in total 27181 magnetic configurations and respective simulated heat load patterns was performed. These two properties have a major impact on the plasma edge and thus the PFC heat load pattern 17 and are closely related to the effect the toroidal plasma current and plasma beta have onto the plasma edge topology [5, 11, 18, 19. The overload evaluation method is applied to this main data set and reveals safe or critical operation regimes for each magnetic configuration in dependence of the convective power onto the PFCs. Such overload maps are highly relevant for the W7-X operation, as they allow to quantify the risk of overload and hint at possible critical states that could be reached by external actuation or occur due to the development of plasma parameters.

In accordance with the line of argumentation presented above, the remainder of this paper is organized as follows. In Section 2 we introduce W7-X and briefly discuss its magnetic field. Section 3 holds the detailed motivation and implementation of the new geometrical models and presents the simulation time speedup introduced thereby. In Section 4 the overload estimation function is introduced and evaluated on the basis of the $t-\Delta R$ scan which is initially described. The paper concludes with a summary and a short discussion of future work which can be pursued on the basis of this work.

\section{The Wendelstein 7-X Mag- netic Field}

W7-X consists of ten half modules. Within each half module, five superconducting non planar coils labeled 1 to 5 are providing the toroidal magnetic flux $\Psi$ as well as the poloidal magnetic flux $\chi$ for a low shear rotational transform profile

$$
t=\frac{d \chi}{d \Psi} .
$$

A finite $t$ is necessary for the compensation of the effects of the $\vec{\nabla} B$ drift. Two further superconducting planar coils (labeled A and B) per half module allow the superposition of a toroidal magnetic flux component. Behind each divertor unit [20] (see Figure 1(a) and Section 3.1, a so-called (island) control coil allows the local alteration of the magnetic field providing the possibility of changing phase and size of the outer magnetic islands. All control coils can be energized independently. Subsequently, only stellarator symmetric fields are considered, which means that the control coils behind the upper and lower divertor respectively are controlled jointly with their counterparts in all modules. The upper and lower control coils are labeled S1 and S2 ("S" referring to the alternative name "sweep coils"). All coils described above are depicted in Figure 1(b)

We normalize the relative winding currents to the relative winding current of the non planar coil 1, i. e.

$$
I_{x}=\frac{n_{x, w} \cdot I_{x, \text { true }}}{I_{n}},
$$

$\forall x \in\{1,2, \ldots, 5, \mathrm{~A}, \mathrm{~B}, \mathrm{~S} 1, \mathrm{~S} 2\}$, where $I_{x, \text { true }}$ corresponds to the current applied to the coils and $n_{x, w}$ denotes the number of windings per coil. In the following, the normalization current $I_{n}$ is equal to $n_{1, w} \cdot I_{1, \text { true }}$. With this representation, the contribution to the overall magnetic field component created by coil type $x$ linearly scales with $I_{x}$.

Nine so-called reference magnetic configurations are defined to represent particular points in the W7-X configuration space 17. They can be arranged in a three-dimensional space of rotational transform $(t)$, radial shift $(\Delta R)$ and mirror ratio $(m)$.

Among these nine reference scenarios, five share similar values of $m$. Changes in the magnetic configuration due to internal plasma currents predominantly take place in two of those dimensions, namely $t$ and $\Delta R$. The mirror ratio can also be changed by internal currents but the magnitude of the change is much smaller than that of $t$ and $\Delta R$.

In leading order, the rotational transform as well as the radial shift are linear combinations of the planar coil currents $I_{\mathrm{A}}$ and $I_{\mathrm{B}}[11$, so for the rest of this paper we shall use normalized versions of $t$ and $\Delta R$, which are identified by $\tilde{t}$ and $\Delta \tilde{R}$ and defined such that

$$
\left(\begin{array}{c}
\Delta \tilde{R} \\
\tilde{t}
\end{array}\right)=\mathbf{R}\left(\begin{array}{c}
I_{\mathrm{A}} \\
I_{\mathrm{B}}
\end{array}\right),
$$

where

$$
\mathbf{R}=\frac{1}{\sqrt{2}}\left[\begin{array}{cc}
-1 & 1 \\
-1 & -1
\end{array}\right]
$$

is the matrix describing a rotation of $\frac{3}{4} \pi$. A high $\tilde{t}$ value corresponds to high rotational transform and 


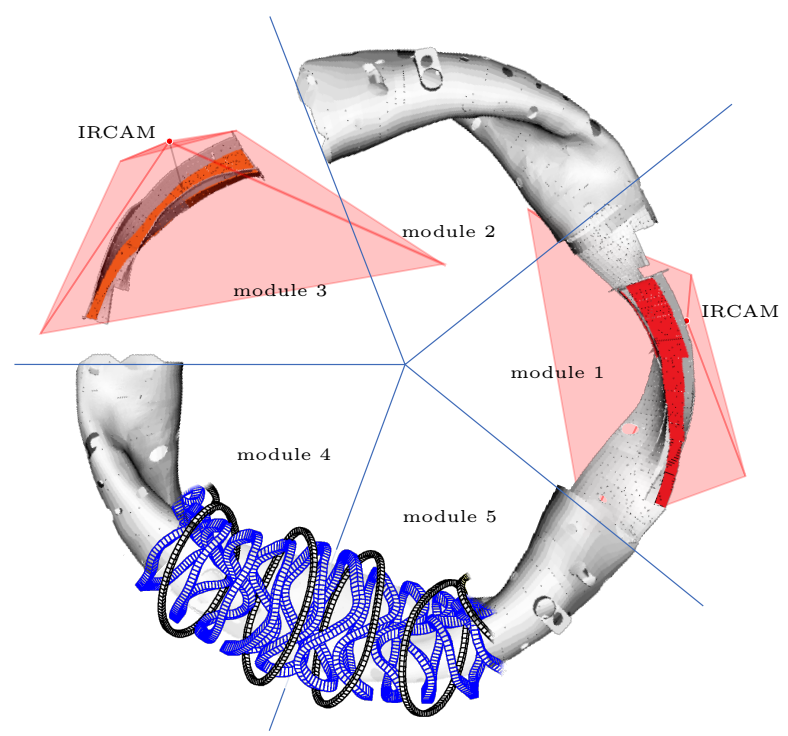

(a) Top-down CAD view of the Wr-X inner vessel, showing the field of view of the IR camera system for the divertor setup. In the cutaway of module 1, the field of view of the IR camera observing an upper divertor is depicted. In module 3 the lower divertor is depicted together with the field of view of the IR camera observing it. One fifth of the total 50 modular (blue) and 20 planar coils (black) is overlaid in modules 4 and 5 .

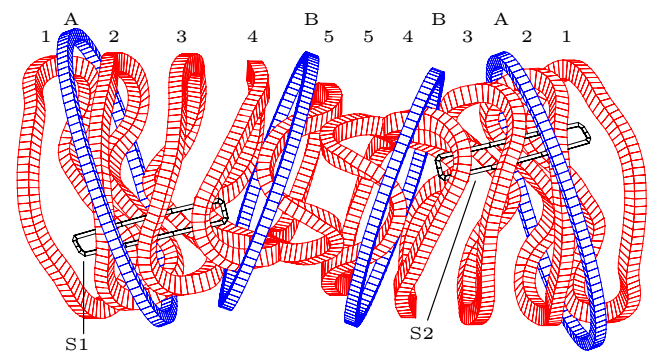

(b) Coils contained in two adjacent half modules with modular coils $1-5$ (red), planar coils A, B (blue) and control coils (black). Each module is symmetric with respect to a radial axis through its center. Adapted from 17 .

Figure 1: Overview of essential parts of $W^{\gamma}-X$ positive $\Delta \tilde{R}$ corresponds to an outward radial shift. Second-order effects are slight deviations especially in the mirror ratio. In the $I_{\mathrm{A}}=-I_{\mathrm{B}}$ axis the value of $m$ is altered slightly due to the opposite sign of $I_{\mathrm{A}}$ and $I_{\mathrm{B}}$, so the inward and outward shifted reference configuration have a small adjustment of the mirror term by means of non-planar coil current adjustments.

\section{Fast and Statistically Signif- icant Convective Heat Load Simulation}

Field line diffusion (FLD) is frequently used to estimate heat loads onto PFCs of magnetic plasma confinement experiments. It is a Monte-Carlo approach where particles are traced along the magnetic field lines. A diffusive behavior is modeled by random displacements in $\left[0, \sqrt{12 D_{\perp} \lambda / v}\right]$ perpendicular to the field lines after a random length $x$ with the distribution $p(x)=\frac{1}{\lambda} \mathrm{e}^{-\frac{x}{\lambda}}$, where $\lambda$ models the mean free path length between two collisions, $D_{\perp}$ is the orthogonal diffusion coefficient and $v$ is the mean particle velocity [21]. The FLD simulation for a particle terminates, when it collides with any PFC. $D_{\perp} / v$ is a physical parameter describing the ratio of perpendicular to parallel transport. Since $D_{\perp}$ actually describes turbulent transport, $\lambda$ must not be identified with a physical mean free path of particles in the plasma edge. Rather it acts as a numerical parameter which must be small compared to the parallel connection length in the scrape-off layer.

Compared with edge transport codes as e. g. EMC3Eirene 22, the FLD model has the advantage of much lower calculation time, whereas the transport process, in particular the dominant anormaleous transport perpendicular to the magnetic field, is simulated in much the same way. Effects occurring at high edge densities like plasma detachment 9 are of course not reflected by FLD simulations.

Assuming a convective power $P_{\text {conv }}$ reaching the PFCs, the convective heat load $q_{i}$ onto the PFC segment $i$, characterized by position and area $A_{i}$ can be calculated as

$$
q_{i}=\frac{n_{i} P_{\text {conv }}}{\zeta n_{\text {tot }} A_{i}},
$$

with $n_{t o t}$ the overall number of hits and the number of hits $n_{i}$ per PFC segment with area $A_{i}$. Symmetries in magnetic fields and PFCs can be utilized to increase the statistics by mapping the hit points onto the smallest symmetric unit. This operation has to be accounted for by introducing $\zeta$ in Equation (5).

In the case of the ideally symmetric W7-X magnetic field and for the PFCs used in the simulation, the assumption of a five-fold toroidal periodicity $\left(q_{i}(\phi, z)=q_{i}(\phi+2 \pi / 5, z)\right)$ describing the W7-X modules, together with a symmetry inherent to each module $\left(q_{i}\left(\phi_{c}+\phi, z\right)=q_{i}\left(\phi_{c}-\phi,-z\right)\right.$, where $\phi_{c}$ is the toroidal angle at the center of each module) is 
justified. This allows for an increase in statistics or, equivalently, the decrease in calculation time by the factor of $\zeta=10$.

On the one hand it is important that the computeraided design (CAD) components resemble the actually installed PFCs appropriately. This means that the CAD surface has to consist of triangles of different sizes which are usually smaller than the demanded spatial heat load resolution on the other hand. In order to retrieve statistically significant heat load patterns onto these triangles, it is usually necessary to start $\mathcal{O}\left(10^{6}\right)$ separate traces which will require a computation time of above ten hour: $\mathrm{n}^{\mathbb{1}}$ per magnetic configuration.

As long as only few heat load simulations are required, this poses no huge problem. However, FLD is a heavily employed tool. FLD applications can mostly be assigned to one ore more of the following categories:

Exploration problem: Magnetic configuration space exploration or FLD parameter scans in the search for magnetic configurations or parameters with particular properties 23,24

Inverse problem: $\quad$ or discharge reconstruction 15 , 25

Forward problem: or discharge planning 26

As motivated before, evolving plasma parameters induce changes in the magnetic configuration and thus dynamic PFC heat loads, which results in the need of multiple simulations for forward problems and even more so for inverse problems. Thus all of the categories above require multiple simulations.

The exploration problem that is going to be introduced in this report requires $\mathcal{O}\left(10^{4}\right)$ different heat load simulations and a rough estimate yields above three years of computation time for one core given the $10^{6}$ separate traces mentioned before ${ }^{\Pi}$. Thus it would be beneficial to reduce the number of necessary traces per configuration without losing statistical significance.

\subsection{PFC Partitioning}

The problem of varying and too small $A_{i}$, accompanied by the need of large simulation times (see last chapter), can be solved together with the question of proper heat load display and analysis. Unfolding the complex 3D geometry onto a 2D surface would have

\footnotetext{
IOptimal performance of a virtual machine cluster with cumulative clock speed of $10 \mathrm{GHz}$ and $16 \mathrm{~GB}$ allocated RAM

IIEach field line trace is an explicit function of the start position and the magnetic field. Thus both parameters can be parallelized. The parallelization of field lines for a given magnetic field is implemented in the 'FIELDLINES' 27] code. In our simulation space the number of available cores is much smaller than both the number of field line traces per configuration as well as the number of configurations, so that no speedup is gained by exploiting the parallelization of both parameters.
}

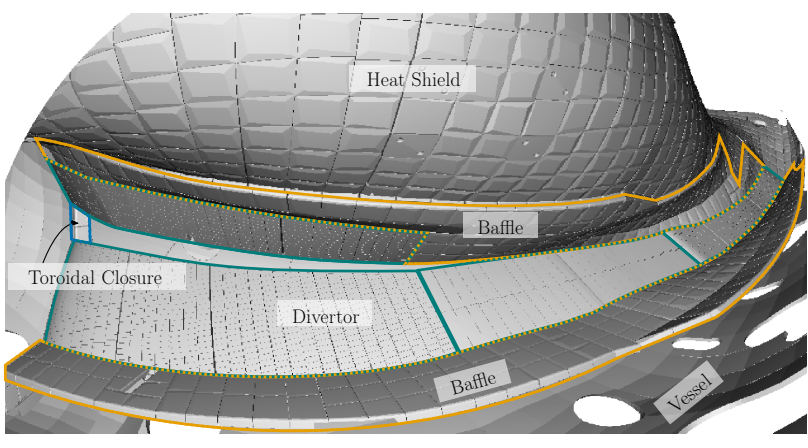

Figure 2: IR camera view of the main loaded Wr-X PFCs

benefits for a variety of applications. For example in ML research groups $2 \mathrm{D}$ rectangular array data as given in images or spectrograms of audio are the preferred input for so-called deep CNNs [28, 29]. To achieve such an architecture, pixel-like input with proper local neighborhood relations is required. Following the current work we aim to apply such CNN architectures to the PFC heat loads. Besides ML applications, the full image processing and analysis tool box can be applied 30].

By splitting and combining triangles of the CAD PFC geometry, both problems are solved. A python library 31] was created with the aim to handle such mesh operations with a focus on operation speed and the abstractness to handle both, simulated FLD hit points as well as experimental heat load patterns.

Divertor Partitioning For W7-X the energy and particle exhaust is realized by an island divertor concept which proved successful in the predecessor experiment Wendelstein 7-AS (W7-AS) 32, 33. Ten divertor target plates are arranged corresponding to the five-fold toroidal symmetry and the up-down flip symmetry inherent to the W7-X device. Every divertor unit is composed of four different parts, labeled and depicted in Figure 3(a). The newly meshed model for the divertor provides a two-dimensional map with well-defined neighborhood relations (see bottom part of Figure 3(a) and significantly larger areas $A_{i}$ as compared to the varying sizes within the original triangulations. Each part of the new segmentation and a corresponding scalar value are represented by one pixel in the newly defined grid and the pixel value, respectively. Pixels that do not have a corresponding counterpart on the divertor are marked white.

The new mesh is inherited from the original mesh and cut into smaller parts, following the natural tile geometry of the target modules (TM) TM1h-4h, TM7h-9h and TM1v-3v (see grey tiles in right half of the zoom in Figure 3(a) . An artificial tile-like substructure is applied to the TM5h-6h target elements (see the left half of the zoom in Figure 3(a) which ensures similar sized areas. The partitioning of the divertor following mainly the tile sizes is also physically motivated 
since the poloidal heat load pattern scale length is on the same order of magnitude as the typical divertor tile width [34]. It may be that for certain plasma scenarios the poloidal heat load pattern scale length will be below the tile size. If so, our method can be extended to smaller partitions. The computational effort would of course go up accordingly. So far, experimental evidence points more to a confirmation of our assumptions than the need for smaller partitions 35 . Since we want to access the heat load $q$ with respect to the maximal design heat load $q_{d, i}$, we furthermore discriminate on the basis of $q_{d, i}$ 36 which is color coded in Figure 3(a) This means in particular that the divertor edges facing the pumping gap between TM1h-4h and TM1v-3v are subdivided along the so-called end top tile and gap tile structures which correspond to lower $q_{d, i}$ of $5 \mathrm{MW}$ and $2 \mathrm{MW}$ respectively (see the right half of the zoom in Figure $3(\mathrm{a})$. The gap tiles are subdivided into two parts parallel to the main target surface (see subdivision of the yellow colored parts in the right half of the zoom in Figure $3(\mathrm{a})$. These cuts are implemented in order to account for the large heat load gradient along this side.

Baffle Partitioning Baffle tiles covering the wall in the vicinity of the divertor are designed to protect the wall from the loads radiated by the divertor plasma. The mapping of the baffle geometry onto a $50 \times 12$ grid, splitting the meshed model along the single baffle tiles, is displayed in Figure 3(b) (cf. Figure 2). In order to guide the eye, the single baffle modules are colored alternatingly. The heat load limit of $0.5 \mathrm{MW} \mathrm{m}^{-2}$ originates from the requirement to keep the temperature of the $\mathrm{CuCrZr}$-structure at the bottom of each tile connecting the fine grained graphite with the cooling pipe below a critical temperature [37. Because of that, the partitioning according to the baffle tile geometry is not only geometrically evident but also motivated from an engineering point of view.

Heat Shield Partitioning The partitioning of the heat shield is motivated and carried out analogous to the baffle partitioning, since the heat shield is constituted from tiles of similar dimension and quality as the baffle tiles (see Figure 2). A corresponding 2D visualization with defined neighborhood relations is not realized yet but easily expandable.

The overload analysis described below is not affected by this complexity reduction. The convective heat load onto the further PFCs is negligible for $P_{\text {conv }}$ up to $10 \mathrm{MW}$.

\subsection{Field Line Diffusion Parameters}

In the following the parameters used for the field line diffusion simulations are motivated. The perpendicular diffusion coefficient $D_{\perp}$ is adjusted to $1 \mathrm{~m}^{2} \mathrm{~s}^{-1}$

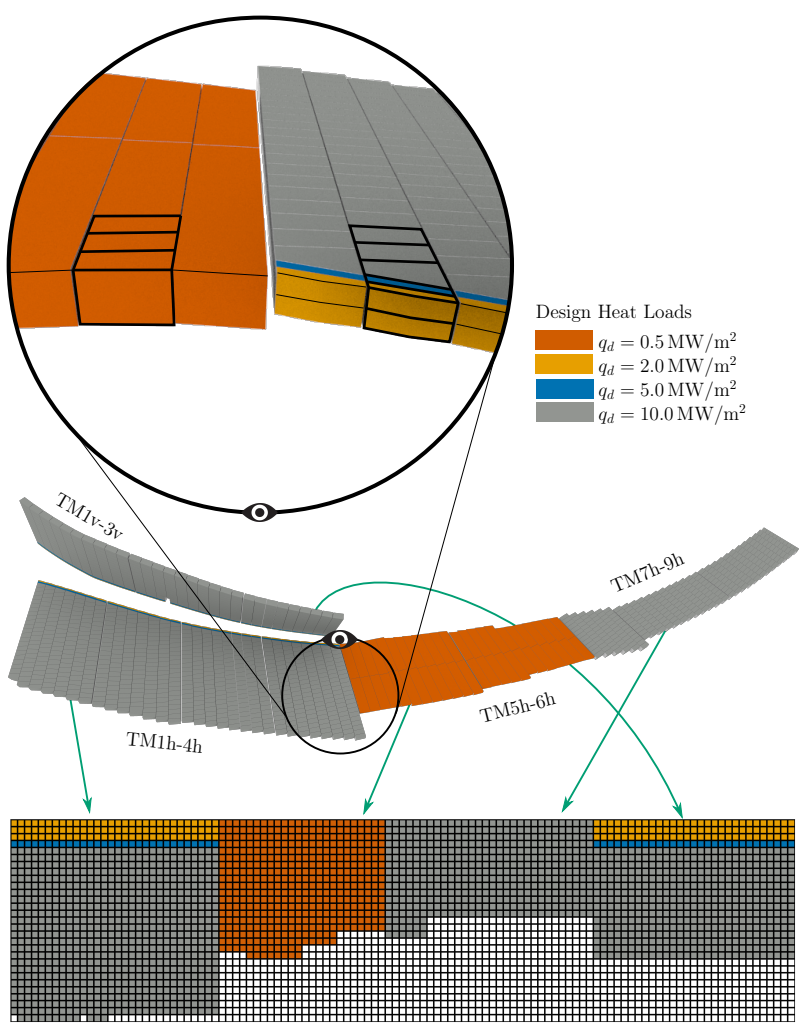

(a) Region definition following the natural tile geometry of the divertor. An artificial tile-like structure is implemented on TM5h-6h ensuring similar segment areas. The top view corresponds to the view of the respective Infra-red camera. Maximum allowed design heat load $q_{d, i}$ is indicated by color. The single segments are sorted into a $113 \times 29$ grid (bottom).

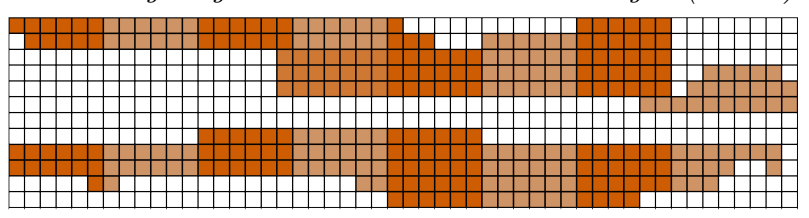

(b) Region definition due to separation of the single baffle tiles into a $50 \times 12$ grid. As indicated by the orange color, the maximum $q$ for the whole baffle is $0.5 \mathrm{MW} \mathrm{m}^{-2}$. The variations in the orange color are highlighting the different baffle modules.

Figure 3: Partitioning the divertor and baffle tiles to a two-dimensional grid with proper neighborhood relations. 
which was encountered in the predecessor experiment W7-AS 38. Evaluation of $D_{\perp}$ for W7-X is currently done using transport calculations in comparison with IR camera measurements and hints to smaller values but of the same magnitude [39].

The average particle velocity at the plasma edge can be approximated as

$$
\begin{aligned}
\langle v\rangle & =\sqrt{\frac{k_{\mathrm{B}}\left(T_{e}+T_{i}\right)}{m_{i}+m_{e}}} \stackrel{m_{i}}{\gtrsim} \underset{m_{e}}{\frac{k_{\mathrm{B}}\left(T_{e}+T_{i}\right)}{m_{i}}} \\
T_{e} \approx T_{i} & \sqrt{\frac{2 k_{\mathrm{B}} T_{e}}{m_{i}}}
\end{aligned}
$$

where the last assumption $T_{e} \approx T_{i}$ is only valid at the edge [5]. With values of $T_{e}=T_{i}=100 \mathrm{eV}$ [5] this results in $v=1.4 \times 10^{5} \mathrm{~m} \mathrm{~s}^{-1}$. A diffusive step perpendicular to a particles field line is evaluated after $\lambda=0.1 \mathrm{~m}$, which is sufficiently small as compared to typical connection lengths (cf. Section 3 .

\begin{tabular}{lr}
\hline \multicolumn{1}{c}{ description } & $q_{d, i}\left[\mathrm{MW} \mathrm{m}^{-2}\right]$ \\
\hline divertor & \\
high loaded area & 10.0000 \\
gap tiles & 5.0000 \\
end top tiles & 2.0000 \\
TM5h-6h & 0.5000 \\
baffle & 0.5000 \\
toroidal closure & 0.5000 \\
heat shield & 0.5000 \\
wall panels & 0.2000 \\
vessel & 0.0019 \\
\hline
\end{tabular}

Table 1: PFCs used for field line tracing. $q_{d, i}$ from 37$]$

Besides the divertor, baffle and heat shield the PFCs included in the FLD process are those installed in the final operational phase (OP2) which are depicted in Figure 2, Together with the local design heat load $q_{d, i}$, they are listed in Table 1. All in-vessel components (IVCs) are designed to withstand an electron cyclotron resonance heating $(\mathrm{ECRH})$ stray radiation power density of $0.05 \mathrm{MW} \mathrm{m}^{-2}$ or are shielded against it [40]. $q_{d, i}$ as well as $A-i$ were chosen in discussion with engineers on the basis of 37] and takes into account failure mechanisms for the component segments represented by our mesh.

\subsection{Heat Load Simulation Speedup}

The remaining field line diffusion parameter to be chosen is $n_{\text {tot }}$. Obviously, $n_{\text {tot }}$ should be sufficiently high in order to achieve a smooth heat load pattern without statistical noise. In other words the calculated heat loads on the segments should no longer show a significant dependence on the choice of $n_{t o t}$. For exactly this reason in the newly partitioned mesh, small segments of the raw mesh are combined with

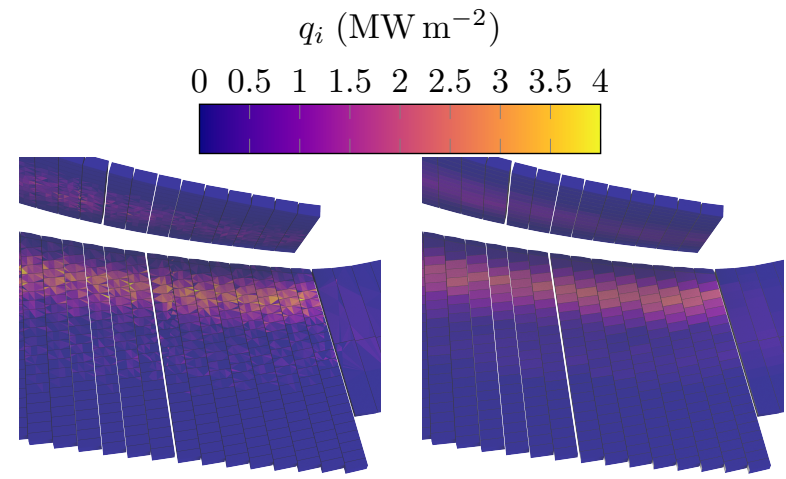

(a) $2.5 \times 10^{4}$ traces on raw mesh

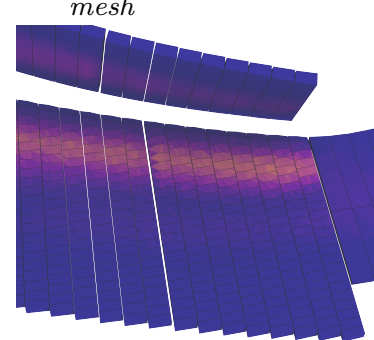

(b) $2.5 \times 10^{4}$ traces on partitioned mesh

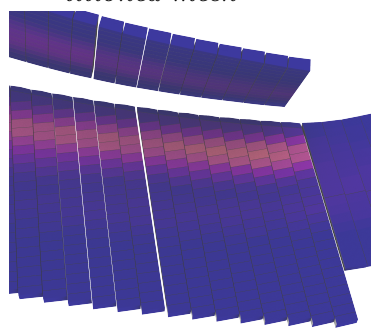

(c) $0.5 \times 10^{6}$ traces on raw mesh

(d) $0.5 \times 10^{6}$ traces on partitioned mesh

Figure 4: Zoom into horizontal part of divertor heat load for simulations of the standard reference case magnetic configuration. The geometrical model (raw vs. partitioned) as well as the total number of field line diffusion traces $n_{\text {tot }}$ changes in between the sub figures.

adjacent segments into the relevant scale size (see Section 3.1).

We perform field line diffusion simulations with ten different $n_{t o t}$ and the parameters introduced in the previous section on the original ("raw") and partitioned mesh. The value of $n_{t o t}=0.5 \times 10^{6}$ shown in Figure $4(\mathrm{c})$ is the commonly used value 41 and results in a smooth pattern. In contrast, the raw heat load pattern for $n_{t o t}=2.5 \times 10^{4}$ has insuficient statistics which leads to a high fluctuation and extreme maximal heat loads (see Figure 4(a) . This is not evident on the partitioned mesh for either $n_{t o t}$ (see Figures $4(\mathrm{~b})$ and $4(\mathrm{~d})$.

To examine the smoothness quantitatively, we examine the fluctuation of the strike-line mean as a function of $n_{t o t}$ normalized to $n_{t o t}=10^{6}$

$$
\frac{\left\langle q_{i}\left(n_{t o t}\right)\right\rangle_{\mathrm{sl}}}{\left\langle q_{i}\left(10^{6}\right)\right\rangle_{\mathrm{sl}}}
$$

The "strike-line" ( $\mathrm{sl}$ ) is defined by those two percent of the total area with the highest heatload. Figure 5 


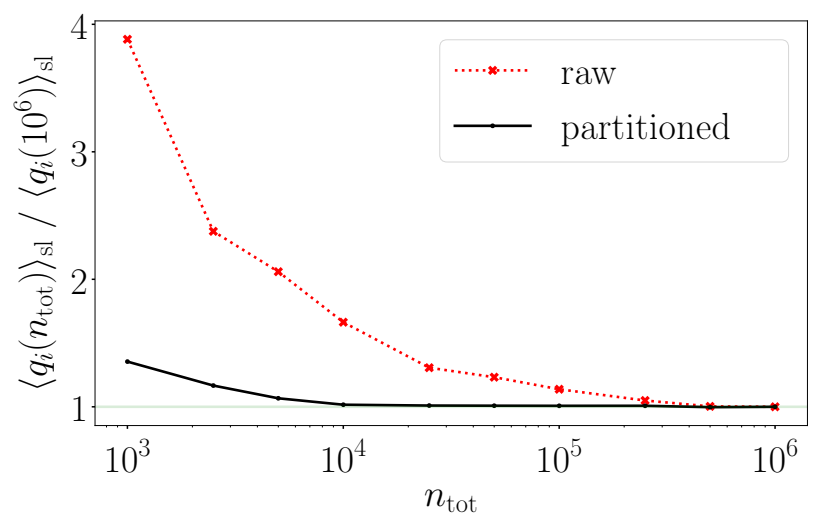

Figure 5: Sensitivity of the average strike-line (sl) heat load with respect to the total number of field line traces $n_{\text {tot }}$ for the original ("raw") or partitioned CAD model (indicated red and black respectively). In order to show convergence the value is nomalized to the reference strike-line at $n_{\text {tot }}=10^{6}$. The data are based on ten independent simulations (varying $n_{\text {tot }}$ ) with the $W^{7-X}$ standard reference magnetic configuration.

demonstrates the convergence of this metric towards unity. As expected, the raw mesh requires a higher $n_{\text {tot }}$ to converge as compared to the newly partitioned mesh. Sufficient convergence can be approximated as $n_{\text {tot }} \geq 0.5 \times 10^{6}$ and $n_{\text {tot }} \geq 2.5 \times 10^{4}$ for the raw and partitioned mesh respectively.

This is also evident in comparing Figures 4(b) and $4(\mathrm{~d})$ where no further improvement is seen with increasing $n_{t o t}$ for the partitioned mesh. The quality of Figure 4(c) is achieved in Figure 4(b) with the partitioned mesh requiring a factor of 20 fewer traces. In conclusion a simulation speed up of a factor of 20 can be reported and hereafter all simulations are performed with $n_{t o t}=2.5 \times 10^{4}$.

\subsection{Heat Load Visualization}

The segmentation technique described in Section 3.1 is demonstrated for various reference configurations in figures 6 and 7 for the divertor and baffle respectively with assumed $5 \mathrm{MW}$ convective power. Well-known patterns as e.g. the standard reference case with the main heat load on TM1h-4h and TM1v-3v [20] can be identified (see left and right quarters of Figure 6(a) respectively).

In the same configuration, the convective heat load onto the baffle as depicted in Figure 7 appears mostly on a baffle tile which is adjacent to the vertical divertor target $(\mathrm{TM} 1 \mathrm{v}-3 \mathrm{v})$.

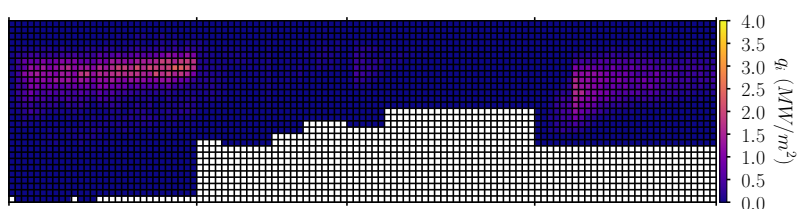

(a) $\left(I_{\mathrm{A}}, I_{\mathrm{B}}\right)=(0.00,0.00)$ i. e. standard reference

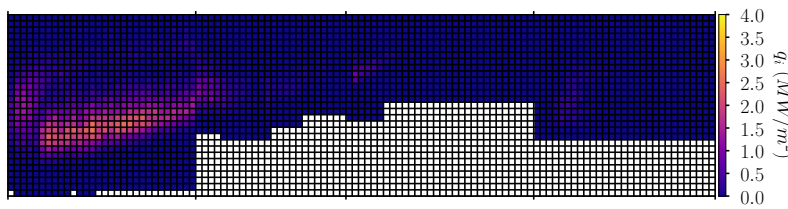

(b) $\left(I_{\mathrm{A}}, I_{\mathrm{B}}\right)=(0.25,0.25)$ i. e. low iota reference

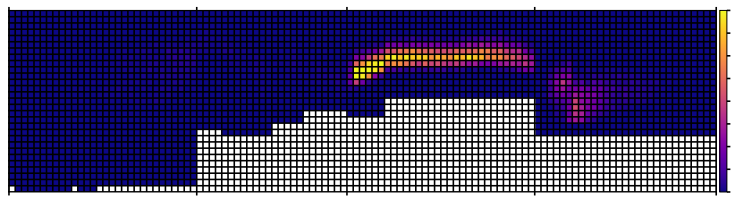

(c) $\left(I_{\mathrm{A}}, I_{\mathrm{B}}\right)=(-0.23,-0.23)$ i. e. high iota reference

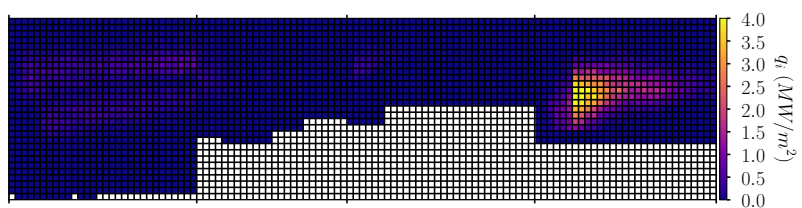

(d) $\left(I_{\mathrm{A}}, I_{\mathrm{B}}\right)=(0.10,-0.20)$ i.e. inward shifted reference

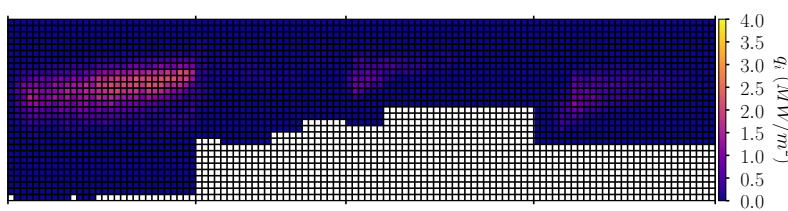

(e) $\left(I_{\mathrm{A}}, I_{\mathrm{B}}\right)=(-0.14,0.14)$ i. e. outward shifted reference

Figure 6: Divertor heat loads visualized in 2D as described in Section 3.1 at $P_{\text {conv }}=5 \mathrm{MW}$

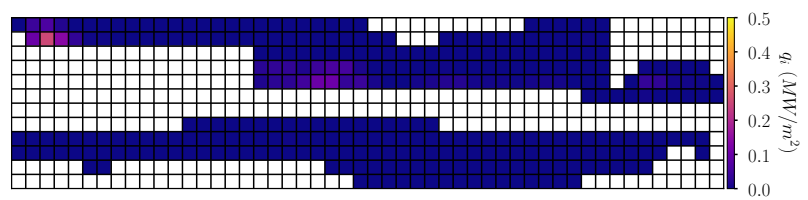

(a) $\left(I_{\mathrm{A}}, I_{\mathrm{B}}\right)=(0.00,0.00)$ i.e. standard reference

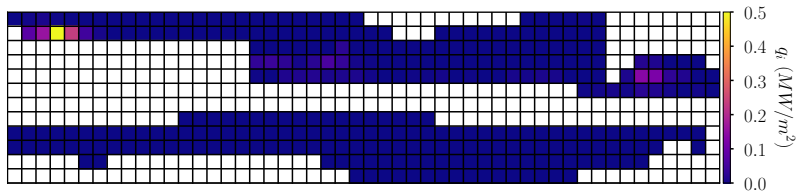

(b) $\left(I_{\mathrm{A}}, I_{\mathrm{B}}\right)=(0.00,-0.10)$

Figure 7: Baffle heat loads visualized in 2D as described in Section 3.1 at $P_{\text {conv }}=5 \mathrm{MW}$ 


\section{Overload Evaluation}

With the speed up in simulation time (cf. Section 3.3) it becomes feasible to perform heat load simulations for magnetic configurations in a narrow spaced two dimensional parameter space with those two plasma parameters affecting the magnetic edge topology the most. Therefore, as motivated before, it is sensible to simulate on the basis of a grid built in the $\tilde{t}-\Delta \tilde{R}$ space (cf. Figure 8) and transformed into the $I_{\mathrm{A}}-I_{\mathrm{B}}$ space according to

$$
\left(\begin{array}{l}
I_{\mathrm{A}} \\
I_{\mathrm{B}}
\end{array}\right)=\mathbf{R}^{-1}\left(\begin{array}{c}
\tilde{t} \\
\Delta \tilde{R}
\end{array}\right) .
$$

The non planar coil currents $I_{1}$ to $I_{5}$ are all set to 1 . The resulting grid is reduced such that $\max \left(\left|I_{\mathrm{A}}\right|,\left|I_{\mathrm{B}}\right|\right) \leq 0.3$, corresponding to the technical limitations of the coil system. Figure 9 depicts the resulting 27181 grid points in the $I_{\mathrm{A}}-I_{\mathrm{B}}$ plane.

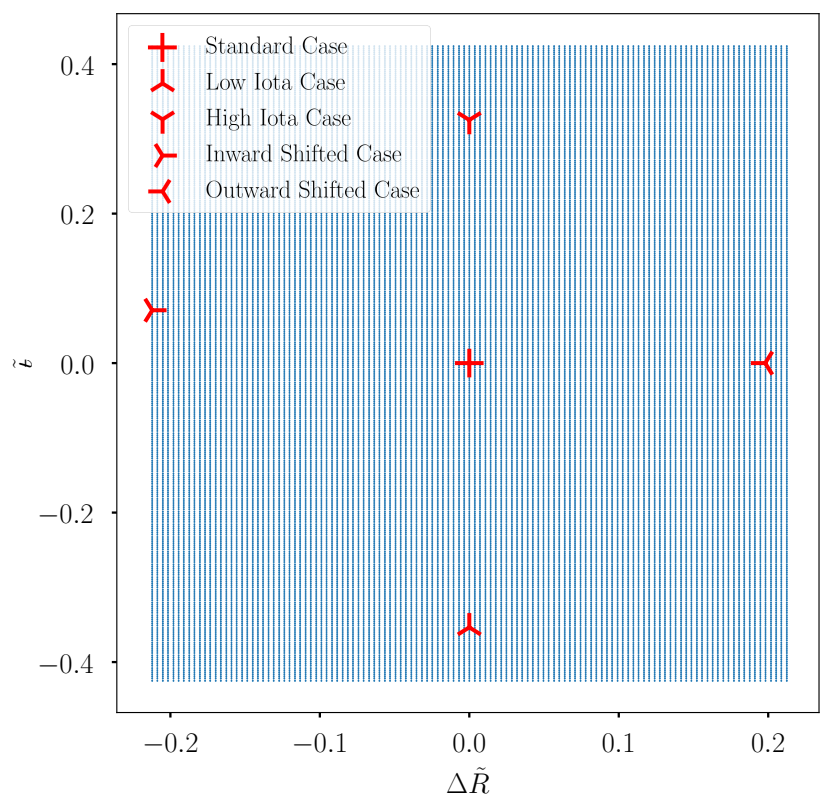

Figure 8: Grid creation with $\Delta \tilde{R}$ : 121 evenly spaced points from $\Delta \tilde{R}=-\frac{0.3}{\sqrt{2}}$ to $\Delta \tilde{R}=\frac{0.3}{\sqrt{2}}$ and $\tilde{t}$ : 301 evenly spaced points from $\tilde{t}=-\frac{0.6}{\sqrt{2}}$ to $\tilde{t}=\frac{0.6}{\sqrt{2}}$, i. e. 36421 grid points. W7-X reference magnetic configurations are indicated by symbols.

\subsection{Overload Definition}

Overload occurs, if any $q_{i}$ exceeds $q_{d, i}$. It is important to consider the noisy character of the field line diffusion simulation and its impact onto the overload, i. e. $q_{i}-$ $q_{d, i}$. With Equation (5) the critical number of hits per area can be calculated as

$$
n_{\text {crit }, i}=\left\lfloor q_{d, i} \frac{\zeta n_{\text {tot }} A_{i}}{P_{\text {conv }}}\right\rfloor
$$

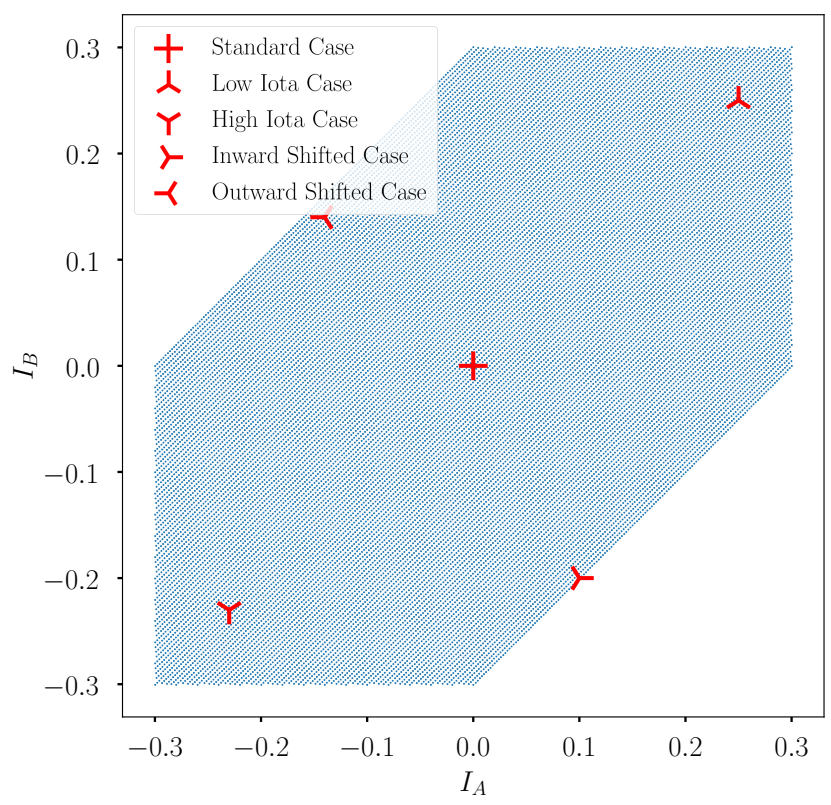

Figure 9: Rotated grid with 27181 points after eliminating points with $\max \left(\left|I_{\mathrm{A}}\right|,\left|I_{\mathrm{B}}\right|\right)>0.3$

where the \lfloor\rfloor operator indicates conservative rounding to the next lower integer.

For independent single Monte-Carlo events, where $n_{i}\left(I_{\mathrm{A}}, I_{\mathrm{B}}\right) \ll n_{\text {tot }}, n_{i}\left(I_{\mathrm{A}}, I_{\mathrm{B}}\right)$ is a good estimate for the rate parameter of a Poisson distribution,

$$
X \sim \operatorname{Poisson}_{n_{i}}(k),
$$

where the random variable $X$ describes the number of hits on segment $i$ in the case of a repeated experiment. The cumulative distribution function is given as

$$
\operatorname{Pr}_{n_{i}}(X \leq k)=\mathrm{e}^{-n_{i}} \sum_{j=0}^{k} \frac{n_{i}^{j}}{j !}
$$

We can utilize Equation (11) to calculate the probability of safe operation (i. e. no overload) for each target element as

$$
\operatorname{Pr}_{n_{i}}\left(X \leq n_{\text {crit }, i}\right)=\mathrm{e}^{-n_{i}} \sum_{j=0}^{n_{\text {crit }, i}} \frac{n_{i}^{j}}{j !}
$$

The varying parameters in Equation $(9)$ are $A_{i}$ and $q_{d, i}$.

With Equation 12, we can combine probabilities for arbitrary PFCs assuming independency:

$$
\operatorname{Pr}\left(X \leq n_{\text {crit }} ; I_{\mathrm{A}}, I_{\mathrm{B}}\right)=\prod_{i} \operatorname{Pr}_{n_{i}}\left(X \leq n_{\text {crit }, i}\right) .
$$

This is what we define as the safety estimation function

$$
\Theta\left(I_{\mathrm{A}}, I_{\mathrm{B}}\right):=\operatorname{Pr}\left(X \leq n_{\text {crit }} ; I_{\mathrm{A}}, I_{\mathrm{B}}\right) .
$$




\subsection{Overload Analysis}

In the following, an evaluation of the probability of safe operation given a certain convective power $P_{\text {conv }}$, i. e. the safety estimation function introduced above (Equation (14) $)$ is presented. The choice of $P_{\text {conv }}$ has to be seen in the context of the planned heating scenarios for OP2 as well as the actual fraction of radiative losses per discharge. For stage I and II total maximum heating powers from $10 \mathrm{MW} \mathrm{W}^{\mathrm{II}}$ to $24 \mathrm{MW} / \mathrm{IV}$ are planned depending on the heating scenario 37 . Recent experiments have demonstrated, that a detached plasma can significantly reduce the convective load onto the divertor by a factor of approximately $10[9]$. This study provides four exemplary values of $P_{\text {conv }}=2,5$, 8 and $10 \mathrm{MW}$ but could be easily extended to other values.

In order to work out the main influences and most critical parts, we shall discuss the different PFCs separately.

\subsubsection{Divertor}

Figure 10 shows the safety evaluation function as a function of $I_{\mathrm{A}}$ and $I_{\mathrm{B}}$ for four different values of $P_{\text {conv }}$ on the basis of the introduced data set. At $P_{\text {conv }}=$ $2 \mathrm{MW}$ a safe operation is apparent. With growing $P_{\text {conv }}$ regions of significant overload i.e. $q>q_{d, i}$ appear and grow.

In the configuration space shown here, the most frequently overloaded part of the divertor is TM5h-6h as shown in Figure 11. It depicts the safety estimation function for each pixel $i$ of the partitioned divertor at $P_{\text {conv }}=5 \mathrm{MW}$ and three different tuples $\left(I_{\mathrm{A}}, I_{\mathrm{B}}\right)$. Four of the five reference configurations contained in the $I_{\mathrm{A}}-I_{\mathrm{B}}$ space simulated (see Figure 9) can safely be run even at $P_{\text {conv }}=10 \mathrm{MW}$ (see Figure 10(d)). The low iota configuration however shows first signs of potential overload at $P_{\text {conv }}=5 \mathrm{MW}$ (see fig. 11(a) and is significantly overloaded at higher $P_{c o n v}$.

\subsubsection{Baffle}

Figure 12 reveals a similar picture for the safety of the baffle. Four of five reference configurations are safe up to $8 \mathrm{MW}$ inclusive. However, the low iota reference configuration is safe for all $P_{\text {conv }}$ whereas the inward shifted case becomes critical in between 5 and $8 \mathrm{MW}$.

\subsubsection{Heat Shield}

The evaluation of the heat shield overload (see Figure 13 reveals some critical load in magnetic fields with high $t$ for a range of outward Shafranov shifts. Furthermore, there is an unsafe region at medium negative $t$ and inward Shafranov shift. However, for the

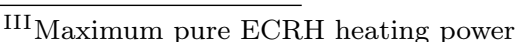

IV Maximum neutral beam injection $(\mathrm{NBI})+$ ion cyclotron radiation heating $(\mathrm{ICRH})$ input power for up to $10 \mathrm{~s}$ in stage II
}

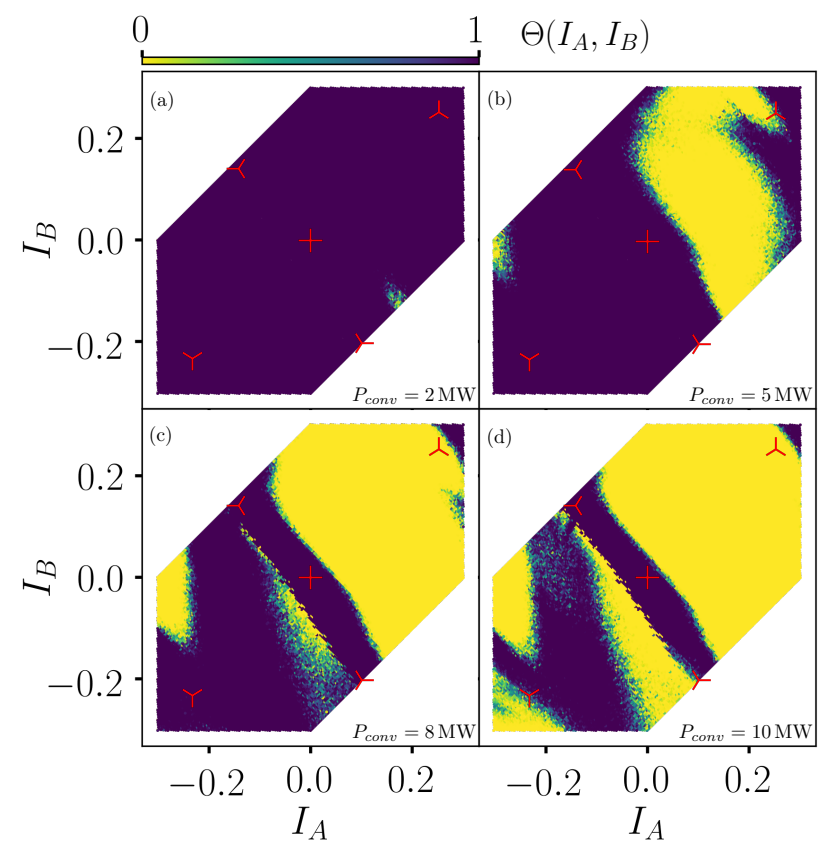

Figure 10: Divertor probability of safe operation for $P_{\text {conv }}=2,5,8$ and $10 \mathrm{MW}$ shown by (a). (d) respectively. The markers indicate five W7-X reference magnetic configurations (see Figure 9).

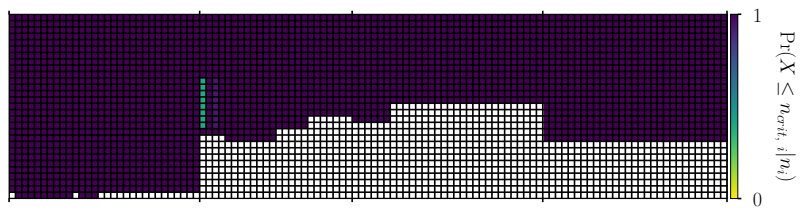

(a) $\left(I_{\mathrm{A}}, I_{\mathrm{B}}\right)=(0.25,0.25)$ i. e. low iota reference

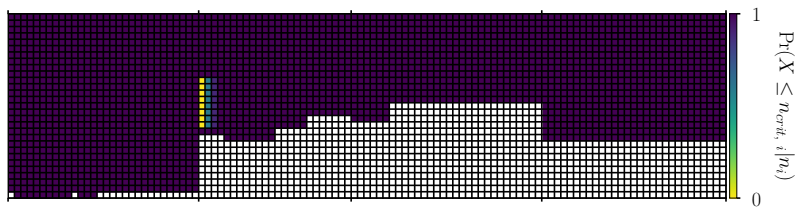

(b) $\left(I_{\mathrm{A}}, I_{\mathrm{B}}\right)=(0.24,0.24)$

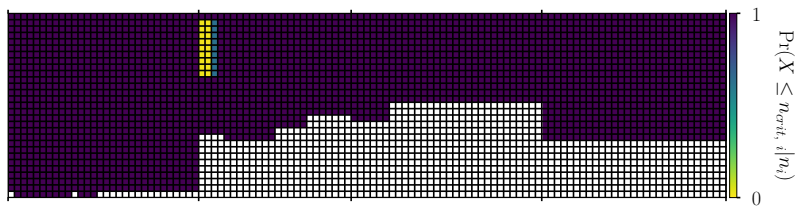

(c) $\left(I_{\mathrm{A}}, I_{\mathrm{B}}\right)=(0.17,0.17)$

Figure 11: Probability for safe operation for three different magnetic configurations. Depicted is the evaluation of $\operatorname{Pr}_{n_{i}}\left(X \leq n_{\text {crit }, i}\right)$ accoring to Equation 12) for each part of the segmented divertor as described in Section 3.1 at $P_{\text {conv }}=5 \mathrm{MW}$ 


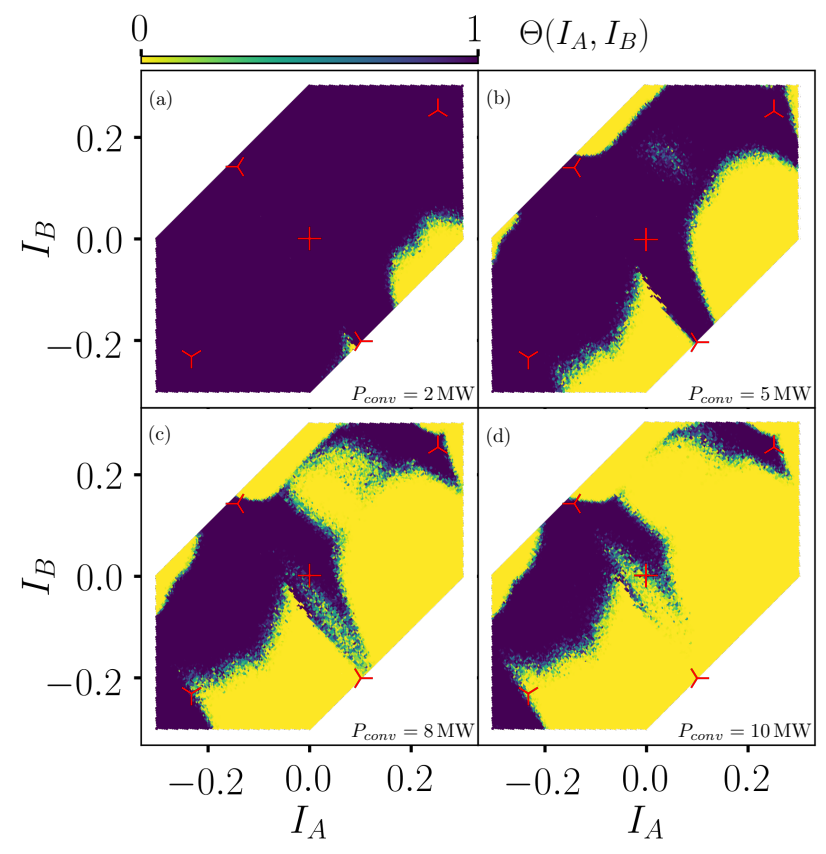

Figure 12: Baffle probability of safe operation for $P_{\text {conv }}=$ 2, 5, 8 and $10 \mathrm{MW}$ shown by $(a)-(d)$ respectively. The markers indicate five $W^{n} 7-X$ reference magnetic configurations (see Figure 9).

convective powers and the parameter space considered, this second region is always a subset of the insecure regions identified for the divertor.

The further components that were included in the FLD simulation, i.e. toroidal closure, wall panels and vessel do not show any critical loads even at $P_{\text {conv }}=10 \mathrm{MW}$. Multiplying the probabilities in the $I_{\mathrm{A}}-I_{\mathrm{B}}$ maps for all PFCs (see Equation (14)), we get a complete picture of the remaining configurations which do not show significant overload risk of any PFC. As an example, Figure 14(a) shows the result for $P_{c o n v}=8 \mathrm{MW}$. The high iota, the outward shifted and the standard reference configurations can be run with no overload on the PFCs at this convective power if plasma effects are negligible.

\subsection{Sweep Coils as Additional Degree of Freedom}

Although W7-X is designed to minimize toroidal currents, their magnitude is rarely zero. Depending on the magnetic configuration and the heating scenario, a toroidal current $I_{\text {tor }}$ converging with the bootstrap current of $\mathcal{O}(10 \mathrm{kA})$ is changing $t$ and thus influences the heat load pattern on the PFCs. In a first approximation, the change of the edge magnetic configuration due to $I_{\text {tor }}$ corresponds to a simultaneous reduction if $I_{\mathrm{A}}$ and $I_{\mathrm{B}}$ toward negatice values $[14]$. This poses a challenge for the operation in the standard configuration at high convective powers as $P_{\text {conv }}=8 \mathrm{MW}$, as can be seen from Figure 14(a), where the orange arrow

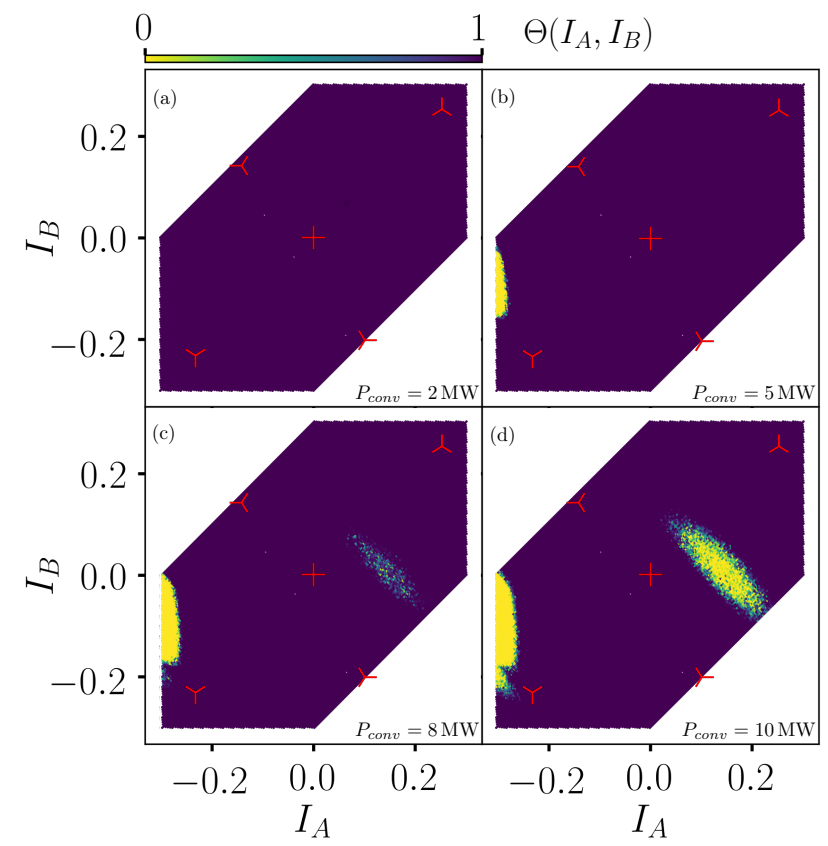

Figure 13: Heat shield probability of safe operation for $P_{\text {conv }}=2,5,8$ and $10 \mathrm{MW}$ shown by $(a)$ (d) respectively. The markers indicate five $W^{r}$-X reference magnetic configurations (see Figure 9).

indicates the path corresponding to a change of $I_{\text {tor }}$ from 0 to $40 \mathrm{kA}$. It is apparent, that such a discharge would potentially damage the divertor as well as the baffle.

Changing the control coil currents $I_{\mathrm{S} 1}$ and $I_{\mathrm{S} 2}$ introduces a third dimension in the configuration space and allows to avoid overload on those PFCs as indicated by the green arrow in Figure 14(b) circumnavigating the critical configuration above the $I_{\mathrm{A}}-I_{\mathrm{B}}$ plane. Such actuators like the control coils can provide additional freedom as demonstrated here.

\section{Conclusion and Future Work}

A redefinition of the $\mathrm{CAD}$ models representing the most important W7-X PFCs has been implemented successfully by merging and splitting faces without loss in geometrical precision. As a result of the model redefinition the required field line diffusion simulation time is reduced by a factor of 20, engineering constraints to the geometry are taken into account and a mapping to unravel the complex 3D PFCs onto 2D rectangular array data can be provided.

The speed-up of the heat load calculation enables simulations based on a fine grid in the rotational transformradial shift parameter space.

A new criterion to evaluate the overload risk has been developed taking statistical fluctuations of the MC based FLD simulation into account. This criterion, 

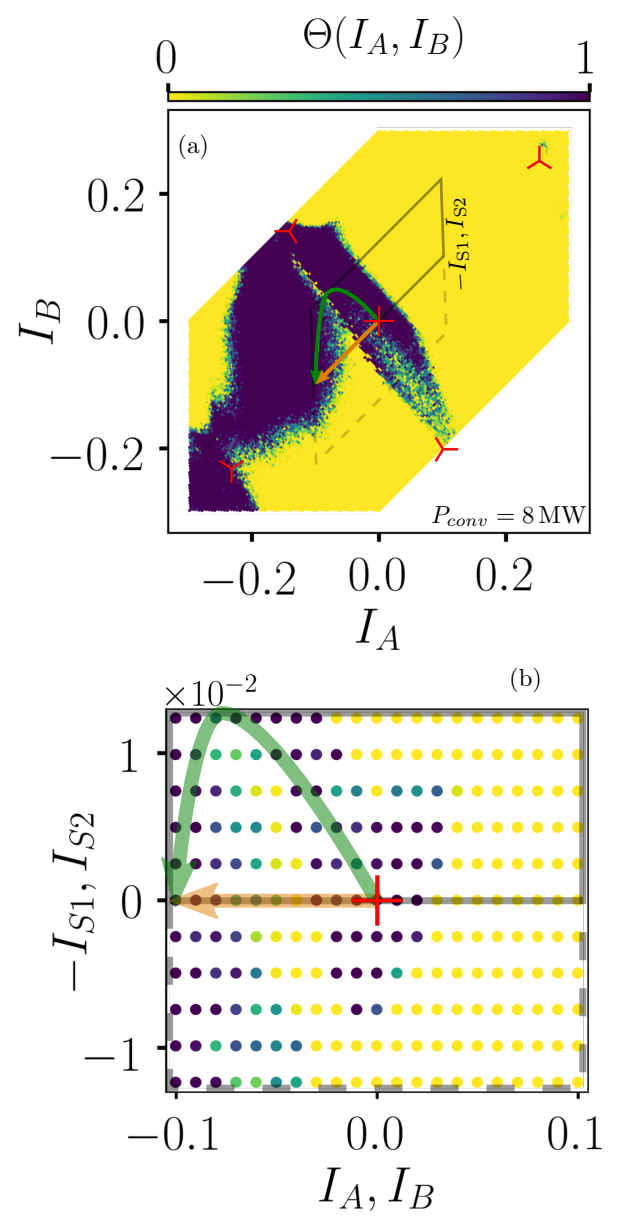

Figure 14: Probability of safe operation for all PFCs and $P_{\text {conv }}=8 \mathrm{MW} .(a)$. Set of magnetic configurations introduced in the introduction of Section 4. (b). Additional data set with control coil variations, which intersects the main data set in the way indicated by grey lines in Figure $14(a)$, The red markers indicate $W{ }^{7}-X$ reference magnetic configurations (cf. Figure 9). The orange arrow indicates $I_{\mathrm{A}}-I_{\mathrm{B}}$ mimicked configurations for $I_{\text {tor }} \in[0,40 \mathrm{kA}]$ (see text) starting from the standard case at $\left(I_{\mathrm{A}}, I_{\mathrm{B}}, I_{\text {tor }}\right)=(0,0,0)$. This corresponds to a path through the configuration space as it would occur due to the time evolution of the toroidal plasma current during the first several $10 \mathrm{~s}$ of a discharge [42]. The green arrow demonstrates a possible route to avoid overload by using the control coils S1 and S2 such that $I_{S 1}=-I_{S 2}$. applied to the simulated data, provides overload maps that will be relevant for machine operation.

These tools developed to improve the heat load analysis constitute a huge improvement for various tasks beyond the applications presented herein. The underlying concepts are applicable to further Monte Carlo based ray tracing simulations, calculating heat loads onto other PFCs without restrictions to the specific W7-X magnetic field and geometry.

In 25, 43 it was shown, that NNs are capable of reconstructing plasma properties from W7-X limiter heat load data. It was proven, that the performance of those networks applied to experimental data was improved by supplementing the training data set with simulations. The present work provides the methods necessary to investigate these findings with the divertor and further PFCs wich is ongoing work. The new visualization mapping is applicable to simulation as well as experimental data and thus provides an interface between the two. The rectangular nature of the new heat load representation allows the implementation of state of the art NN architectures as for example CNNs, and beyond this gives access to all kinds of image manipulation and analysis tools.

As a long term objective we pursue heat load pattern control in W7-X for which this work constitutes a highly relevant building block. We envision reinforcement learning approaches which require reward functions in order to have a quantity to optimize for 44 45. The overload maps found in Section 4 could be used as such.

\section{Acknowledgments}

This work has been carried out within the framework of the EUROfusion Consortium and has received funding from the Euratom research and training programme 2014-2018 and 2019-2020 under grant agreement No 633053. The views and opinions expressed herein do not necessarily reflect those of the European Commision.

We wish to acknowledge the helpful discussions with M. Endler, L. Rudischhauser, J. Fellinger, J. Geiger, R. Labahn and T. Sunn Pedersen.

\section{Bibliography}

[1] H.-S. Bosch et al. "Technical challenges in the construction of the steady-state stellarator Wendelstein 7-X". In: Nuclear Fusion 53.12 (2013), p. 126001. ISSN: 0029-5515.

[2] J. Nührenberg et al. "Overview on Wendelstein 7-X Theory". In: Fusion Technology 27.Suppl. S (1995), pp. 71-78. 
[3] T. Sunn Pedersen et al. "Confirmation of the topology of the Wendelstein 7-X magnetic field to better than 1:100000". In: Nature Communications 7 (2016), p. 13493. ISSN: 2041-1723.

[4] T. Klinger et al. "Performance and properties of the first plasmas of Wendelstein 7-X". In: Plasma Physics and Controlled Fusion 59.1 (2017), p. 014018. ISSN: 0741-3335.

[5] A. Dinklage et al. "Magnetic configuration effects on the Wendelstein 7-X stellarator". In: Nature Physics (2018), pp. 1-6. ISSN: 17452481.

[6] C. Beidler et al. "Physics and Engineering Design for Wendelstein VII-X". In: Fusion Science and Technology 17.1 (1990), pp. 148-168.

[7] J. Nührenberg and R. Zille. "Stable stellarators with medium $\beta$ and aspect ratio". In: Physics Letters A 114.3 (1986), pp. 129-132. ISSN: 03759601.

[8] Thomas Sunn Pedersen et al. "Key results from the first plasma operation phase and outlook for future performance in Wendelstein 7-X". In: Physics of Plasmas 24.5 (2017), p. 055503. ISSN: 10897674.

[9] Thomas Sunn Pedersen et al. "First results from divertor operation in Wendelstein 7-X". In: Plasma Physics and Controlled Fusion 61.1 (2019), p. 014035. ISSN: 0741-3335.

[10] V. Erckmann, U. Gasparino, and H. Maaßberg. "Current drive and bootstrap current in stellarators". In: Plasma Physics and Controlled Fusion 34.13 (1992), pp. 1917-1923. ISSN: 0741-3335.

[11] P. I. Strand and W. A. Houlberg. "Magnetic flux evolution in highly shaped plasmas". In: Physics of Plasmas 8.6 (2001), pp. 2782-2792. ISSN: $1070664 \mathrm{X}$.

[12] Y Feng et al. "Impact of Island Geometry on Island Divertor Performance". In: Koch, R.; Lebedev, S.: 30th EPS Conference on Plasma Physics and Controlled Fusion, European Physical Society (2003) (2003).

[13] M. Jakubowski et al. "Development of infrared and visible endoscope as the safety diagnostic for steady- state operation of Wendelstein 7-X". In: QIRT 100 (2014).

[14] H. Hölbe et al. "Access to edge scenarios for testing a scraper element in early operation phases of Wendelstein 7-X". In: Nuclear Fusion 56.2 (2016), p. 026015. ISSN: 0029-5515.

[15] Y. Gao et al. "Approaches for quantitative study of divertor heat loads on W7-X". In: 14th Quantitative InfraRed Thermography Conference. 2018, pp. 6-7.

[16] Achim Klenke. Probability theory : a comprehensive course. 2014. ISBN: 1848000472.
[17] T. Andreeva. Vacuum magnetic configurations of Wendelstein $7-X$. Tech. rep. IPP-III/270. Greifswald: Max-Planck-Institut für Plasmaphysik, 2002 .

[18] J Geiger et al. "Physics in the magnetic configuration space of W7-X". In: Plasma Physics and Controlled Fusion 57.1 (2015), p. 014004. ISSN: 0741-3335.

[19] Per Helander. "Theory of plasma confinement in non-axisymmetric magnetic fields". In: Reports on Progress in Physics 77.8 (2014), p. 087001. ISSN: 0034-4885.

[20] Hermann Renner et al. "Physical Aspects And Design of the Wendelstein 7-X Divertor". In: Fusion Science and Technology 46.2 (2004), pp. 318-326.

[21] S.A. Bozhenkov et al. "Service oriented architecture for scientific analysis at W7-X. An example of a field line tracer". In: Fusion Engineering and Design 88.11 (2013), pp. 2997-3006. ISSN: 09203796.

[22] Y. Feng et al. "Recent Improvements in the EMC3-Eirene Code". In: Contributions to Plasma Physics 54.4-6 (2014), pp. 426-431. ISSN: 08631042.

[23] P. Sinha et al. "Numerical studies of scrapeoff layer connection length in Wendelstein7-X". In: Nuclear Fusion 58.1 (2018), p. 016027. ISSN: 0029-5515.

[24] F. Effenberg et al. "Numerical investigation of plasma edge transport and limiter heat fluxes in Wendelstein 7-X startup plasmas with EMC3-EIRENE". In: Nuclear Fusion 57.3 (2017), p. 036021.

[25] D. Böckenhoff et al. "Reconstruction of Magnetic Configurations in W7-X using Artifical Neural Networks". In: Nuclear Fusion 58.5 (2018), p. 056009.

[26] J. D. Lore et al. "Modeling and preparation for experimental testing of heat fluxes on W7-X divertor scraper elements". In: IEEE Transactions on Plasma Science 46.5 (2018), pp. 1387-1392. ISSN: 00933813.

[27] Samuel A. Lazerson et al. "First measurements of error fields on W7-X using flux surface mapping". In: Nuclear Fusion 56.10 (2016), p. 106005. ISSN: 0029-5515.

[28] Yann Lecun, Yoshua Bengio, and Geoffrey Hinton. "Deep learning". In: Nature 521.7553 (2015), pp. 436-444. ISSN: 14764687. arXiv: arXiv:1312. 6184v5. 
[29] Jürgen Schmidhuber. "Deep learning in neural networks: an overview." In: Neural networks : the official journal of the International Neural Network Society 61 (2015), pp. 85-117. ISSN: 1879-2782. arXiv: 1404.7828.

[30] J. R. Parker. Algorithms for Image Processing and Computer Vision. 2nd ed. Wiley, 2011, p. 481. ISBN: 978-0-470-64385-3.

[31] Daniel Böckenhoff and Marko Blatzheim. tfields: numpy + sympy implementation of tensor fields with attached coordinate systems. https://pypi.org/project/tfields/. 2018.

[32] P. Grigull et al. "First island divertor experiments on the W7-AS stellarator". In: Plasma Physics and Controlled Fusion 43.12A (2001), A175-A193. ISSN: 0741-3335.

[33] K. McCormick et al. "Island divertor experiments on the Wendelstein 7-AS stellarator". In: Journal of Nuclear Materials. Vol. 313-316. SUPPL. 2003, pp. 1131-1140. ISBN: 0022-3115.

[34] M Endler et al. "Managing leading edges during assembly of the Wendelstein 7-X divertor". In: Plasma Physics and Controlled Fusion 61.2 (2019), p. 025004. ISSN: 0741-3335.

[35] H. Niemann et al. "Large wetted areas of divertor power loads at Wendelstein 7-X". In: Physical Review Letters (2019), To be published.

[36] J. Boscary et al. "Design improvement of the target elements of Wendelstein 7-X divertor". In: Fusion Engineering and Design 87.7-8 (2012), pp. 1453-1456. ISSN: 09203796.

[37] R. Brakel et al. Specification of design loads for in-vessel components of $w^{r} 7-x$. Tech. rep. 1-ACS0005.1. Max-Planck-Institut für Plasmaphysik, 2017, p. 57.

[38] F. Wagner et al. "Overview on W7-AS Results with Relevance for Wendelstein 7-X and the Low-Shear Stellarator Line". In: Fusion Energy Proc. 17th Int. Conf. Yokohama: IAEA Vienna, CD-Rom file and https://inis.iaea. org / collection / NCLCollectionStore / \{_ Public/31/011/31011276.pdf, 1998, pp. 115128.

[39] F. Effenberg et al. "Investigation of 3D effects on heat fluxes in performance-optimized island divertor configurations at Wendelstein 7-X". In: Nuclear Materials and Energy 18 (2019), pp. 262267. ISSN: 23521791.

[40] R. Brakel et al. Technische Richtlinie Einsatz von Materialien und Verwendung von magnetisierbaren Werkstoffen an Wr-X. Tech. rep. 1NBT-T0010.12. 2017.

[41] M. Endler et al. Private Communication. 2019.
[42] Arnold Lumsdaine et al. "Design and analysis of the W7-X divertor scraper element". In: $F u$ sion Engineering and Design 88.9-10 (2013), pp. 1773-1777. ISSN: 09203796.

[43] Marko Blatzheim et al. "Neural network performance enhancement for limited nuclear fusion experiment observations supported by simulations". In: Nuclear Fusion 59.1 (2019), p. 016012. ISSN: 0029-5515.

[44] R. Sutton and A. Barto. Reinforcement Learning: An Introduction. Vol. 3. 9. 1998, p. 360. ISBN: 0262193981. arXiv: 1603.02199 .

[45] Timothy P. Lillicrap et al. "Continuous Control with Deep Reinforcement Learning". In: International Conference on Learning Representations 2016 (2016). arXiv: 1509.02971 